09-11 July 2021

Paris, France

\title{
Electronic absorption spectra of nickel (II) complexes used for sensitized solar cells: DFT/TDDFT Study
}

\author{
Sefia BRAHIM \\ Department of chemistry, University of Saida, Algeria
}

\begin{abstract}
A detailed theoretical study of electronic absorption spectra of $\mathrm{Ni}$ (II) complexes in a distorted square planar form. Such complexes have been the goal of several researches due to their special properties in many aspects; they are investigated in terms of their pertinence for use in DSSCs. We investigated the electronic structures and UV-Vis spectra of $\mathrm{Ni}$ (II) complexes with density functional theory (DFT) and time-dependent density functional theory (TDDFT) calculations with CAM-B3LYP and PBE0 hybrid functional. Moreover, the analysis of the frontier molecular orbitals (FMOs) which allowed a detailed assignment to interpret the electronic transitions of the calculated spectral properties. The influences of functional, substitution and solvent on electronic spectra have been assessed. According to the computed results, the geometry optimization was in good agreement with the experimental results from $\mathrm{X}$-ray diffraction. The theoretical calculations reproduce the main observed spectroscopic proprieties and substituent effects of $\boldsymbol{e c d a}$ and $\boldsymbol{d t c}$ ligands on the absorption spectra.

As experimentally observed, computations results reveal that all complexes show a tree absorption bands in UV region and very low absorption one in visible region. We have assigned these bands to the electronic transitions answerable for their appearance; they are characterized by mixed character mainly dominated by MLCT and LLCT character.
\end{abstract}

Keywords: DSSC; Excitation; metal; theoretical; transition. 